\author{
Prof. Dr. Dominik Irnich \\ Interdisziplinäre Schmerzambulanz, Klinik für Anaesthesiologie, \\ LMU Klinikum der Universität München, Campus Innenstadt \\ Pettenkoferstraße 8 A, 80336 München, Deutschland \\ dominik.irnich@med.uni-muenchen.de
}

\title{
Akupunktur online?
}

Liebe Leserinnen und Leser,

als die Ausgangsbeschränkungen kamen, haben wir lange überlegt, ob wir unsere Fort- und Weiterbildungskurse online anbieten sollen. Natürlich gab es Kontakte zu allen befreundeten Fachgesellschaften und wir waren alle der Meinung: Nein!

Eine Akupunkturausbildung ist eine praktische Ausbildung, wir brauchen den Kontakt zwischen Lehrendem und Lernendem, die Nadel muss gefühlt werden, in der praktischen Übung vom Akupunkteur und vom Akupunktierten. Akupunkturausbildung braucht Praxis.

Webinar und Onlinemeeting sind nichts Neues und bereits vor 10 Jahren kamen Angebote großer Verlage für E-LearningPlattformen. Da ist vieles ins Leere gelaufen und weitergehende Akzeptanz fehlte. Noch 2017 wurde E-Learning als gescheitert erklärt, auch weil es nicht die Strukturen gab (siehe Andreas Wittke, Hochschulforum Digitalisierung). Aber die Digitalisierung schritt voran, Blended und Binge Learning, MOOCS, "flipped-classroom", Gruppenchats bei WhatsApp wurden entwickelt. Entschuldigen Sie die Anglizismen, aber ohne geht es wohl nicht in der digitalisierten Welt.

Mit der Krise und den Ausgangsbeschränkungen entstanden auch in der Akupunkturszene neue Bedürfnisse. Und so sind auch wir den Weg gegangen und haben nach 34 Jahren das erste Mal die Internationale Akupunkturwoche online angeboten, natürlich verdaulich in Seminaren von 90-180 min. Es war ein riesiger Erfolg und wurde großartig angenommen (siehe Verbandsseiten DÄGfA).

Akupunkturkurse zur Zusatzbezeichnung wird es auch in Zukunft nur begrenzt online geben, aber es gibt ja noch viel mehr TCM zu lernen. Und alle Verfahren der TCM haben theoretische Inhalte, die auch online vermittelt werden können.

Aber was sind die Voraussetzungen für eine gute Onlinelehre? Wir müssen unterscheiden zwischen Lehrenden und Lernenden. Lehrende brauchen eine gute technische Ausrüstung, didaktische Planung, Kreativität, Bereitschaft zur Innovation und Zeit für die Vorbereitung. Einfaches Streaming einer aufgezeichneten Vorlesung oder eines Kurses ist eigentlich obsolet. Die Lernenden müssen sich ebenfalls neuen Herausforderungen stellen. Das Video einschalten, damit der Lehrende nicht in eine schwarze Wand spricht, sollte eine Selbstverständlichkeit sein. Aber es ist auch Mit-

Deutsche Zeitschrift für Akupunktur 2020 • 63 (3): 121

https://doi.org/10.1007/s42212-020-00305-0

๑) Springer Medizin Verlag GmbH, ein Teil von Springer Nature 2020 arbeit erforderlich. Lebendige Lehre besteht aus Fragen und Diskussionen, Mitarbeit in Kleingruppen und Teilnahme an Onlineselbsttests. Dazu sollte jeder bereit sein, der etwas lernen möchte.

Idealerweise gibt es für einen Kurs bereits vorab Material zum Selbststudium. Der Dozent steht zu Beginn für Fragen und Diskussionen bereit. Dann geht es in Kleingruppen, in denen das Wissen am praktischen Fall angewendet wird. Das Ergebnis wird dann wieder im großen Klassenzimmer vorgestellt und diskutiert. Entscheidend für das Lernen eines umfassenden Themas mit vielen Unterrichtseinheiten ist die Taktung und Betreuung in den Zwischenphasen. Einbau von intelligenten tutoriellen Systemen kann in diesen Phasen auch ein weiteres Eigenstudium erlauben, am besten begleitet von virtuell vernetzten Kleingruppen. An vielen Hochschulen werden gerade entsprechende und weitergehende Konzepte umgesetzt und getestet.

Es ist eine spannende Zeit in der Lehre und dennoch funktioniert alles nur, wenn Begeisterung und Enthusiasmus für das Fachgebiet vorhanden sind.

Und was ist mit den Nebenwirkungen? Ja, die gibt es. Die Schlafstörungen nach ganztägigen Onlineveranstaltungen sind ausreichend beschrieben. Wir warten auf weitere Meldungen. Analog denken sei erlaubt. Nach 2,5 h täglich Social-Media-Nutzung steigt der Grad der Unzufriedenheit, aber vielleicht sind hier andere Faktoren wirksam. Wir werden die Folgen genau beobachten müssen.

Ganz gleich, welche spannenden und lehrreichen Erfahrungen wir noch vor unserem Laptop, Handy, Pad oder PC in Zukunft machen werden, wir brauchen den persönlichen Austausch, das dreidimensionale authentische Erleben, das Handanlegen und die vielen Gerüche und Geschmäcker der Traditionellen Chinesischen Medizin.

Das taktile Spüren und Blättern der aktuellen $D Z A$ in unseren Händen ist eine weitere sensorische Dimension des Austausches und des Lernens, die wir auf keinen Fall aufgeben dürfen. Viel Spaß beim Lesen und Blättern!

Ihr

Dominik Irnich

Interessenkonflikt. D. Irnich gibt an, dass er Aufwandsentschädigungen für Lehr- und Vortragstätigkeit von öffentlichen Institutionen und gemeinnützigen Vereinigungen erhält und in seiner Funktion als 1. Vorsitzender und Leiter des Fortbildungszentrums der DÄGfA. 